\title{
Correspondence
}

\section{Injury to renal vein during fixation of fractured lumbar spine and subsequent atrophy of kidney in a female patient with spinal cord injury and paraplegia}

Spinal Cord (2003) 41, 527-529. doi:10.1038/sj.sc.3101484

We report a rare complication of surgical management of fracture of lumbar spine.

\section{Case report}

A 48-year-old female patient with bipolar affective disorder attempted to commit suicide by falling from a bridge. She sustained comminuted fractures of calcanei, burst fracture of the body of L-3 with virtual obliteration of the spinal canal at this level by the large displaced posterior segments and paraplegia (sensory level L-2; ASIA grade A). Decompression and fusion were carried out by a left side retroperitoneal approach through the bed of 12th rib. During the operative procedure, considerable venous bleeding occurred when the kidney and pararenal fat were reflected. Bleeding was controlled with packs. While mobilising the kidney, the left kidney appeared to be of normal size and shape. The L-3 vertebral fragments and adjacent discs were excised and stabilisation was carried out with a Synthes jacking cage and supported with a Ventrofix between L-2 and L-4. At the end of the procedure, when the packs were removed there was again copious venous bleeding. Injury to renal vein was then recognised and damage at two areas of renal vein was repaired. The patient made a satisfactory postoperative recovery and was transferred to spinal injuries centre after 12 weeks.

The patient underwent global assessment in the spinal unit. Intravenous urography (IVU) was performed. IVU revealed no excretion of contrast on the left side (Figure 1). Tomogram showed marked atrophy of left kidney (Figure 2). The right kidney had a normal outline; pelvicalyceal system and ureter were normal. Ultrasound examination of left kidney revealed no hydronephrosis. The kidney measured $5.0 \mathrm{~cm}$ (Figure 3). No flow was detectable on colour flow Doppler. A small amount of blood flow was seen on the power Doppler settings (Figure 4). The right kidney showed normal blood flow. The ultrasound appearances were those of atrophy of left kidney. Blood biochemistry: urea: $8.0 \mathrm{mmol} / 1$; creatinine: $86 \mu \mathrm{mol} / 1$. Blood pressure was $111 / 54 \mathrm{mmHg}$. At present, this patient has a long-term indwelling urethral catheter for drainage of urinary bladder.

\section{Discussion}

Complications, which can occur during anterior surgical approach to lumbar spine, may be classified as vascular, visceral, muscular, urogenital, or neural. ${ }^{1}$

- Vascular complications: Injury to renal vessels, radicular vessels, iliolumbar vein, ascending lumbar vein complex.

- Visceral complications: Splenic rupture, hepatic injury, bowel perforation, peritoneal tears, intussusception, superior mesenteric artery syndrome, atelectasis, pneumonia, pulmonary embolism, postoperative ileus, pneumo/haemothorax, chylothorax, and acute cholecystitis.

- Muscular complications: Injury to diaphragm, psoas muscle, and haematoma of latissimus dorsi along its lateral aspect.

- Urogenital complications: Operative injury to kidney, and ureter, trauma to hypogastric plexus leading to retrograde ejaculation or infertility in men. Delayed hydronephrosis due to retroperitoneal fibrosis and ureteral stricture has been reported. ${ }^{2}$ Furthermore, mechanical correction of severe curves and aggressive positioning may cause angulation of the ureter with hydronephrosis. ${ }^{3}$

- Trauma to lumbar plexus: In the case of a complete lesion of the lumbar plexus, the patient would present with paresis and atrophy in the distribution of the femoral and obturator nerves: weakness of thigh flexion, leg extension, thigh eversion, and thigh adduction. Sensation may be lost over the inguinal region, genitalia, and the lateral anterior and medial thigh. The patellar and cremasteric reflex may be lost on that same side as well. ${ }^{1}$

\section{- Injury to lumbar sympathetic chain.}

In this patient, trauma to left renal vein occurred at two places during fixation of lumbar spine fracture. Although damage to renal vein was repaired, follow-up IVU showed no excretion of contrast by the left kidney. Ultrasound scan confirmed marked atrophy of left kidney. Probably, repair of renal venous tear at two places led to occlusion or thrombosis of renal vein, or there was inadvertent trauma to renal artery as well during surgical control of copious bleeding from the renal vein. We used power flow Doppler ultrasound 


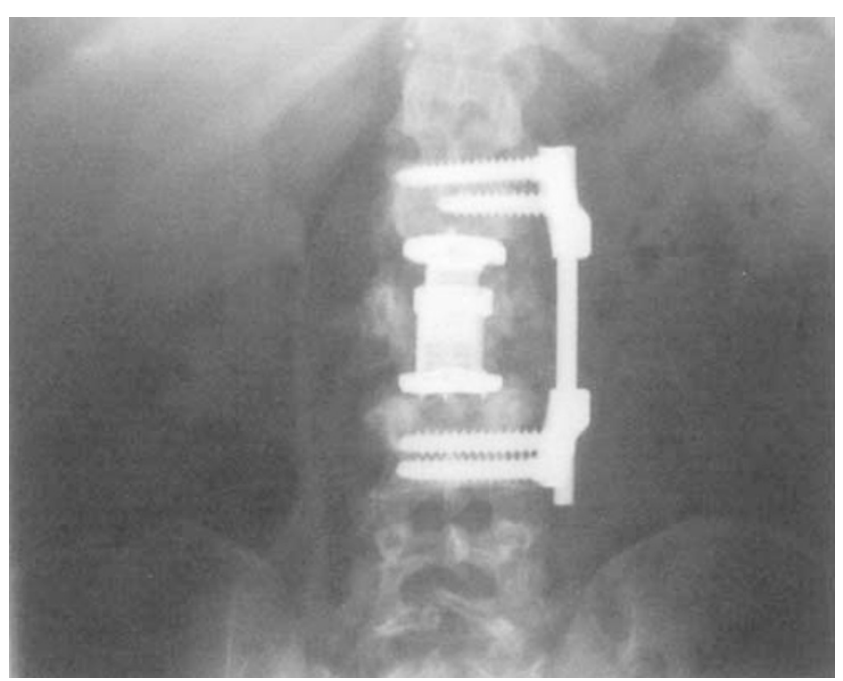

Figure 1 A 5 min film of intravenous urography shows excretion of contrast by right kidney. However, there is no excretion of contrast by the left kidney. Spinal fixation system is seen

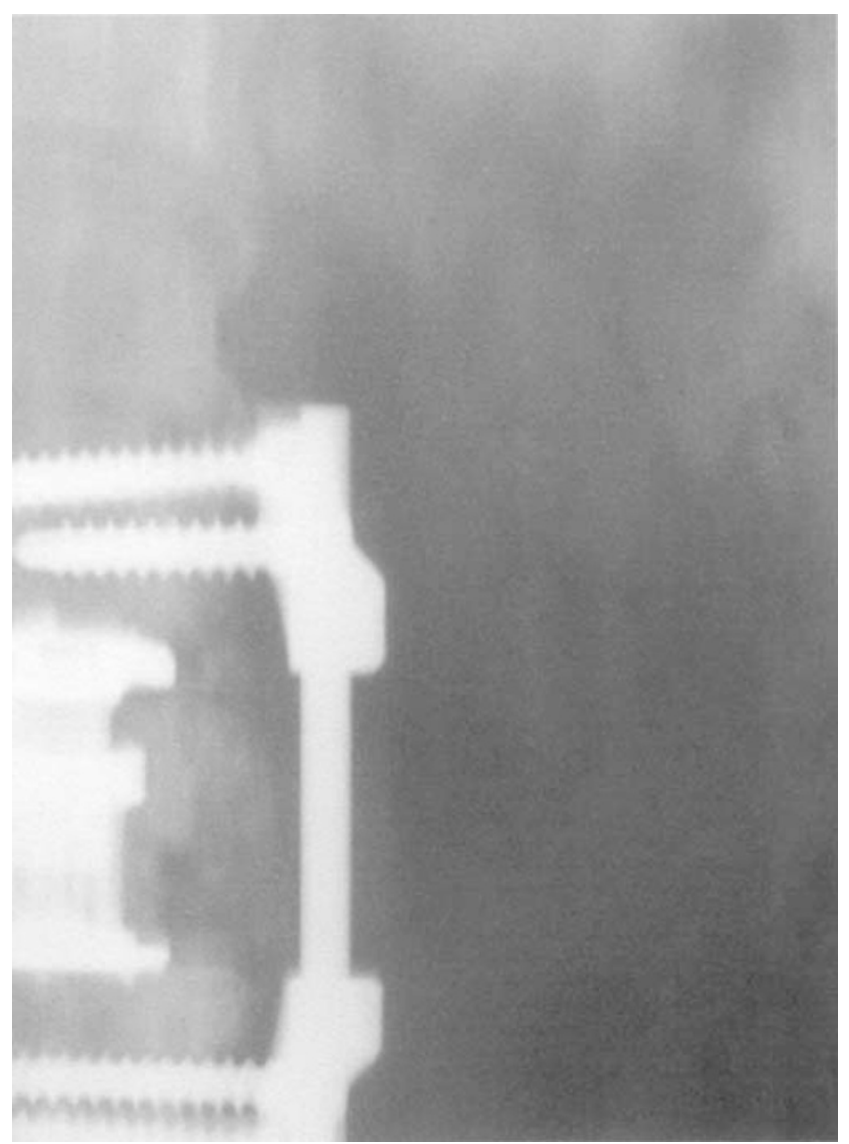

Figure 2 Tomogram of intravenous urography shows marked atrophy of left kidney

imaging to assess perfusion of left kidney. Power flow imaging (PFI), in contrast to colour flow imaging (CFI), is based on the integrated power spectrum. The hue and

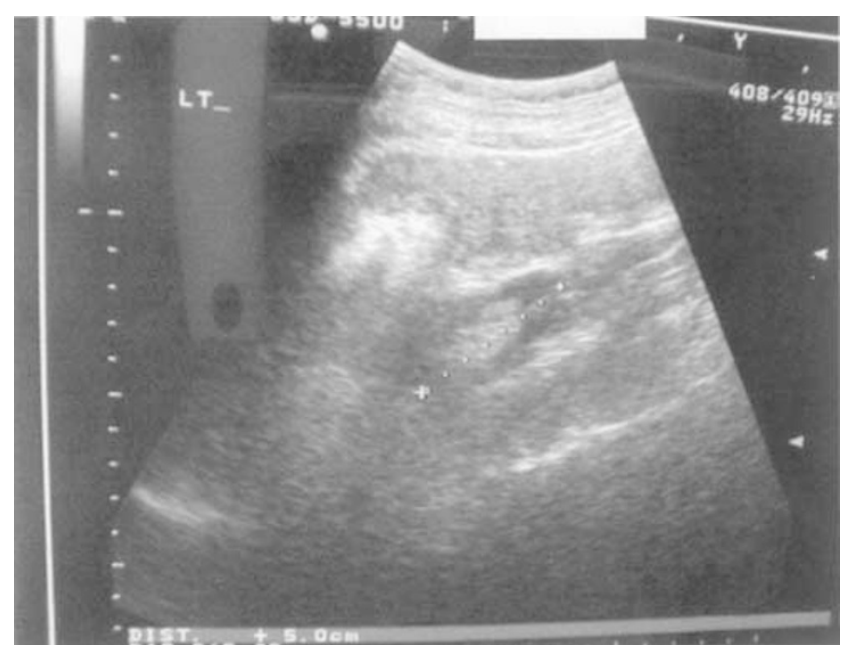

Figure 3 Ultrasound scan of left kidney reveals no hydronephrosis. Left kidney measures $5 \mathrm{~cm}$

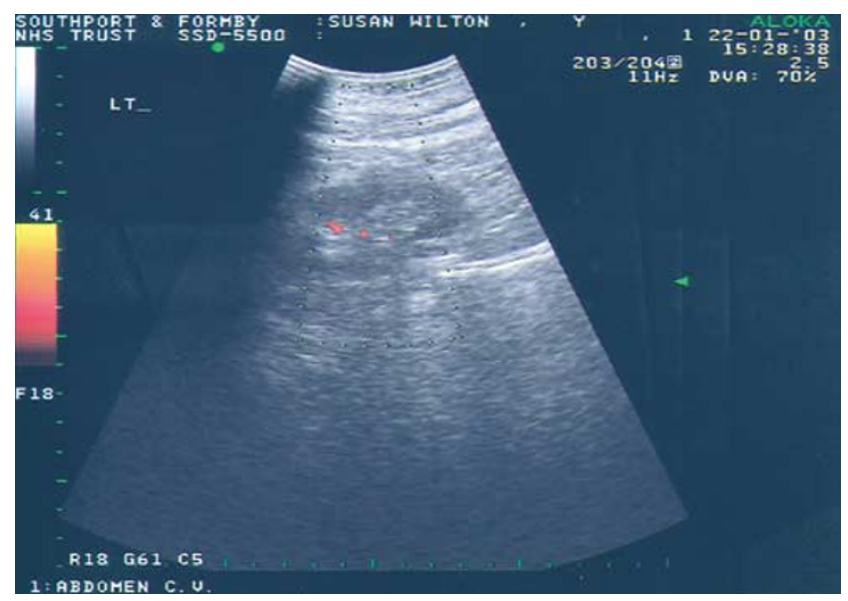

Figure 4 Power flow Doppler ultrasound shows reduced blood flow in left kidney

the brightness of the PFI colour signal reflects the pressure amplitude of the Doppler-shifted acoustic signal, which is directly related to the quantity and the acoustic impedance of the flowing blood. Significant advantage of this technique is uniformly low power of background noise. As a result, PFI gain can be more enhanced than the CFI gain before acoustic noise begins to obscure the flow imaging, a very useful method to improve sensitivity substantially for low flow rates. In addition, the technique is almost angle independent, which is another advantage especially in the evaluation of minimal residual blood flow as in the case of atrophic kidney with decreased renal perfusion. Overhoff et $a l^{4}$ demonstrated reduced parenchymal perfusion in transplanted kidneys by power Doppler ultrasound, as PFI is highly sensitive to slow flow velocities. 
Division of left renal vein may not always be innocuous. During surgery for aortic aneurysm, division of left renal vein resulted in a significant increase in serum creatinine level. The left renal vein was divided in $28(8 \%)$ of the 355 patients undergoing elective aneurysm resection. The mean immediate postoperative creatinine values were significantly higher after left renal vein division, $193 \pm 174 \mu \mathrm{mol} / 1, \quad$ compared to $133 \pm 93 \mu \mathrm{mol} / 1$ for those whose left renal vein remained intact ( $P$ less than 0.05$)$. The left renal vein was divided in $17(14 \%)$ of the 123 patients having emergency surgery for ruptured aortic aneurysm, $61(49 \%)$ of whom survived more than 30 days. The mean immediate postoperative creatinine values were significantly higher after left renal vein division, $426 \pm 277 \mu \mathrm{mol} / 1$, compared to those in whom the vein was left intact, $178 \pm 136 \mu \mathrm{mol} / 1$ ( $P$ less than 0.05 by Mann-Whitney $U$-test). After 1 month, serum creatinine levels were still significantly higher in those patients in whom the left renal vein had been divided. The authors of this study from Department of Vascular Surgery, Royal Prince Alfred Hospital, Sydney, Australia, concluded that although division of the left renal vein was a useful way to improve exposure of the juxtarenal aorta, the manoeuvre was associated with an adverse effect on renal function. ${ }^{5}$

Patients, who sustained trauma to lumbar spine, may be referred to spinal injuries centre after they have undergone surgical fixation of lumbar spine. Knowledge of possible complications, which may occur due to surgical fixation of lumbar spine, will help spinal cord clinicians to modify the rehabilitation programme and follow-up schedules to suit the clinical condition of each patient with lumbar spine fracture. For example, recurrent renal calculi in spinal cord injury patients with a single kidney carry risks for decreasing renal function, urosepsis, and death. ${ }^{6}$ Spinal cord injury patients with congenital anomalies of urinary tract require a close follow-up. ${ }^{7}$

\author{
S Vaidyanathan ${ }^{1}$, P Hughes ${ }^{2}$, S Sett $^{1}$, T Oo $^{1}$, BM Soni ${ }^{1}$ \\ and G Singh ${ }^{1}$ \\ ${ }^{1}$ Regional Spinal Injuries Centre, District General \\ Hospital, Town Lane, Southport, Merseyside, UK; and \\ ${ }^{2}$ Department of Radiology, District General Hospital \\ Town Lane, Southport, Merseyside, UK
}

\section{Acknowledgements}

The authors are grateful to Mr. Patrick Tierney, Senior Medical Representative, Shire Phrmaceuticals Ltd, Chineham, Basingstoke, Hampshire, United Kingdom and Coloplast Ltd, Peterborough, United Kingdom for sponsoring the printing of colour illustration.

\section{References}

1 Samudrala S, Khoo LT, Rhim SC, Fessler RG. Complications during anterior surgery of the lumbar spine: an anatomically based study and review. Neurosurg Focus 1999; 7: http:/www.medscape.com/viewarticle/413590_4

2 Cleveland RH, Gilsanz V, Lebowitz RL, Wilkinson RH. Hydronephrosis from retroperitoneal fibrosis after anterior spine fusion. A case report. J Bone Joint Surg Am 1978; 60: 996-997.

3 Keim HA, Weinstein JD: Acute renal failure - a complication of spine fusion in the tuck position. A case report. J Bone Joint Surg Am 1970; 52: 1248-1250.

4 Overhoff HM, Mutze S, Teistler M, Ehrhardt J. Quantification of renal partial volumes from 3D-reconstruction of power Doppler ultrasound scans. http://www.umi.cs.tu-bs.de/ full/archive/niere/

5 Huber $\mathrm{D}$ et al. Does division of the left renal vein during aortic surgery adversely affect renal function? Ann Vasc Surg 1991; 5: 74-79.

6 Stover SL, Wiggins KC. Single kidney outcome and management in persons with spinal cord injury. $J$ Spinal Cord Med 2000; 23: 2-5.

7 Vaidyanathan S et al. Klippel-Feil syndrome - the risk of cervical spinal cord injury: a case report. BMC Fam Pract 2002; 3: 6 . 